\title{
Usos crossmedia y cross-promotion en la difusión de contenidos de los diarios nativos digitales europeos
}

\author{
José Sixto García \\ Universidad de Santiago de Compostela \\ jose.sixto@usc.es \\ https://orcid.org/0000-0002-2988-0975
}

\author{
Xosé López García \\ Universidad de Santiago de Compostela \\ xose.lopez.garcia@usc.es \\ https://orcid.org/0000-0002-1873-8260
}

\section{Crossmedia and cross-promotion uses in the dissemination of content in European digital native newspapers}

\begin{abstract}
RESUMEN ABSTRACT
Desde hace aproximadamente una década se empezó a hablar de convergencia periodística como el proceso de integración de formas de comunicación tradicionalmente separadas que afecta a las empresas, a las tecnologías, a los profesionales y a los públicos tanto en la producción como en la distribución y el consumo de contenidos. Los periodistas han comenzado a elaborar productos que se distribuyen a través de plataformas diversas para poder adaptarse a las necesidades de consumo de las audiencias. En esta investigación analizamos el proceso de adaptación de los cibermedios nativos digitales europeos a las estrategias crossmedia a partir de una muestra compuesta por cinco cabeceras europeas: eldiario.es (España), Mediapart. fr (Francia), observador.pt (Portugal), niusdiario.es (España) y De Correspondent (Holanda). Para ello se emplea una metodología de carácter exploratorio y se recurre al análisis estadístico de datos en redes sociales y aplicaciones. Los resultados determinan implementación de narrativas

Journalistic convergence is the process of integrating traditionally separate forms of communication that affects companies, technologies, professionals and audiences in production, distribution and consumption. of content. Journalists have started to develop products that are distributed through various platforms according to the consumption needs of audiences. In this research, we analyze the process of adaptation of European digital native media to crossmedia strategies based on a sample of five European media: eldiario.es (Spain), Mediapart.fr (France), observador.pt (Portugal), niusdiario.es (Spain) and De Correspondent (Holanda). For this, an exploratory methodology and statistical analysis of data in social networks and applications are used. The results determine the implementation of crossmedia narratives and adaptation of the contents to the different screens and supports, as well as the detection of transmedia practices that force the user to complement the informative storytelling on different platforms.
\end{abstract} crossmedia y adaptación de los contenidos a las diferentes pantallas y soportes, así como la detección de prácticas transmedia que obligan al usuario a complementar el storytelling informativo en plataformas diferentes.

PALABRAS CLAVE

Cibermedios nativos digitales; Crossmedia; Cross-content; Cross-promotion; Redes sociales; Apps.

\section{KEYWORDS}

Native digital media; Crossmedia; Cross-content; Cross-promotion; Social networks; Apps. nativos digitales europeos. Hipertext.net, (21), 141-151. https://doi.org/10.31009/hipertext.net.2020.i21.13 


\section{Introducción}

Cada cierto tiempo surge un debate acerca de la capacidad para renovar el periodismo de las empresas de comunicación y de los avances tecnológicos (García Avilés, 2006). Uno de los más destacados y transcendentes de la historia reciente de la comunicación se originó en la década de 1990 y comienzos de la siguiente en relación con los procesos de convergencia, que empezaban a materializarse en algunos medios bajo la esfera de una sociedad en red que comenzaba a asentarse (Castells, 2000). Poco a poco los medios fueron abocados a ceder el paso ante los nuevos hábitos y demandas informativas de los públicos del siglo XXI (Salaverría y Negredo, 2008) provocadas por la digitalización y apresuradas por circunstancias coyunturales como la situación económica y estructurales como el alejamiento de las audiencias hacia los medios tradicionales.

En España los procesos de concentración de redacciones llegaron más tarde que en otros países, en especial Estados Unidos, donde a mediados de los años 90 las empresas periodísticas comenzaron a configurarse como conglomerados multimedia y multiplataforma, no solo desde el punto de vista económico. De todas formas, como las redacciones integradas permiten atender las demandas de unas audiencias cada vez más multiplataforma y resultan más baratas fueron surgiendo diferentes iniciativas de procesos de convergencia, que a finales de la década de 2000 ya se habían convertido en tendencia. Ligados fundamentalmente a la convergencia tecnológica, fueron liderados por empresas de telecomunicaciones, productores de dispositivos informáticos y desarrolladores de aplicaciones digitales (Salaverría, 2010).

No obstante, los medios de comunicación experimentaron procesos de convergencia desde mucho antes de que el impacto de la tecnología digital produjera una aceleración e intensificación del proceso (De Sola Pool, 1990) y está claro que hoy en día transcienden la integración de redacciones. Salaverría, García Avilés y Masip (2010) definen la convergencia periodística como "un proceso multidimensional que, facilitado por la implantación generalizada de las tecnologías digitales de telecomunicación, afecta al ámbito tecnológico, empresarial, profesional y editorial de los medios de comunicación, propiciando una integración de herramientas, espacios, métodos de trabajo y lenguajes anteriormente disgregados, de forma que los periodistas elaboran contenidos que se distribuyen a través de múltiples plataformas, mediante los lenguajes propios de cada una" (2010, p. 59).

Es cierto que en un primer momento la convergencia fue entendida como producto, designando únicamente el proceso de confluencia de tecnologías sin contemplar ni la configuración empresarial ni el perfil de los periodistas (Negroponte, 1979; Goolding, Murdock, 1996). Más tarde, asimilado el concepto de convergencia como resultado de la confluencia de tecnologías, varios investigadores aludieron a su carácter sistémico (Singer, 2004; Gordon, 2003; Jenkins, 2004, 2006) y contemplaron no solo los aspectos instrumentales, sino otros ámbitos como la producción o el consumo de los medios de comunicación. Finalmente, en una tercera etapa, la convergencia se entendió como un proceso sujeto a gradación, como un continuum (Dailey, Demo y Spillman, 2010) compuesto por diferentes fases que dan como resultado final la convergencia. Así, por ejemplo, el modelo propuesto por Dailey y sus colaboradores incluye cinco fases: la promoción coordinada, el clonado, la competencia mutua, compartir contenidos y convergencia.

En la teoría de la convergencia periodística se pueden diferenciar cuatro grandes modelos en base al área organizacional a la que incumben:

- La convergencia tecnológica como condición previa y necesaria a cualquier proceso de convergencia porque permite que los contenidos digitales puedan ser fácilmente distribuidos a través de diferentes plataformas (Masip y Micó, 2009).

- La convergencia empresarial que posibilita diversificar el riesgo empresarial, ampliar el negocio en nuevos sectores y aprovechar las sinergias en los ámbitos diferentes como la publicidad, la producción, la documentación, la distribución o la promoción (Doyle, 2002; Salaverría, García Avilés y Masip, 2010).

- La convergencia profesional ligada a la voluntad de las empresas periodísticas de acelerar el flujo de los contenidos informativos a través de diferentes canales para obtener un mayor beneficio económico (Jenkins, 2006), lo que implica que los periodistas trabajen conjuntamente para producir productos destinados a plataformas diferentes con la finalidad de alcanzar audiencias masivas en una escala 24/7 (Quinn, 2005).

- Gordon (2003) se refirió a la convergencia de contenidos en relación al modelo de convergencia narrativa (storytelling) que los periodistas polivalentes deberían producir en función de las posibilidades multimedia e interactivas que ofrecen las tecnolo- 
gías para la producción y el consumo, de manera que las audiencias pueden acceder a una gran variedad de contenidos actualizados permanentemente desde distintos dispositivos y plataformas.

Así pues, hoy en día la convergencia periodística se concibe como un proceso de integración de los modos de comunicación tradicionalmente separados que afecta a las empresas, a las tecnologías, a los profesionales y a las audiencias en todas las fases de la producción, la distribución y el consumo de contenidos. Por tanto, la convergencia incide en las diferentes esferas de la ocupación periodística, es decir, en las estrategias empresariales, en los cambios tecnológicos, en la elaboración y distribución de contenidos multiplataforma, en el perfil profesional de los periodistas y en las formas de acceso a los contenidos (Salaverría, García Avilés y Masip, 2010). Entender o limitar, pues, la convergencia a una o parte de los ámbitos mencionados implica una visión reduccionista que no refleja la complejidad de los procesos periodísticos y empresariales actuales.

\section{Crossmedia, cross-promotion y transmedia en el escenario de la convergencia}

En el contexto de la convergencia tecnológica, empresarial, profesional y de contenidos el periodismo adapta los modelos crossmedia o cross-content implementados hasta entonces en el marketing. Sustentadas en un nuevo sistema de emisión multiplataforma que puede basarse en un modelo de comunicación de masas personalizada debido a que los nuevos medios digitales permiten a los usuarios decidir qué contenidos recibir, cuándo y de qué manera (Villa, 2011), las prácticas crossmedia se caracterizan por integrar diferentes canales, soportes o medios para lanzar un mensaje común, pero adaptado a las diferentes normas y lenguajes específicos. En este sentido, los modelos crossmedia siempre exigen interactuar con el usuario para que participe en cada una de las presencias que tenga el medio en redes o aplicaciones móviles, por lo que es posible que las audiencias salten de una plataforma a otra sin abandonar la marca. En realidad, se trata de emplear de forma cruzada todos los medios posibles para promocionar una presencia en Internet, lo que también implica entender los hábitos conductuales de los nuevos usuarios, que, acostumbrados a moverse de un sitio a otro, abandonaron su rol exclusivo de consumidores pasivos para implicarse en la producción y difusión de contenidos (prosumers) (Martínez-Sala, Segarra-Saavedra y Monserrat-Gauchi, 2018), llegando incluso a convertirse en prescriptores de las marcas (adprosumers) (Martínez-Sala y Campillo-Alhama, 2018; Segarra-Saavedra y Tur-Viñes, 2017). De este modo, al establecerse expe- riencias cross-content los medios garantizan que si un usuario declina un producto o un formato, se le presente otro alternativo y relacionado.

En realidad, los modelos crossmedia suponen una forma de integrar contenidos de medios cruzados o convergentes para proporcionar a los usuarios información complementaria (Ma, Tanaka y Nadamoto, 2006). Los medios se han visto obligados a aceptar ciertos cambios en sus rutinas productivas como consecuencia de la convergencia debido a la cantidad de actores que están ansiosos por competir en el mercado y que están capacitados para hacerlo en entornos virtuales, por lo que asumir estos desafíos y adaptarse a la evolución tecnológica resulta determinante para la gran mayoría de las empresas de comunicación (Franquet y Villa, 2012; Kjus, 2014). Ibrus y Scolari (2012) describen estos modelos como una propiedad intelectual, servicio, historia o experiencia distribuida a través de múltiples plataformas mediáticas usando diversos formatos.

En la mayor parte de casos los procesos de convergencia también adoptaron formas de producción cruzada o cross-promotion, que se convirtieron en una herramienta muy poderosa (Copple, 2012), hasta el punto de que para Ketterer et al. (2004) este tipo de convergencia es la única que funciona. Como se trata de usar textos o recursos audiovisuales para publicitar contenidos producidos y distribuidos por un medio asociado (Dailey, Demo y Spillman, 2010), no suelen suponer la creación de contenidos específicos para cada medio, al menos significativamente distintos. Según Lowrey (2005), la promoción cruzada es la forma de convergencia más común en Estados Unidos e investigaciones como la de Tang, Newton y Wang (2007) concluyeron que el empleo de las promociones cruzadas coordinadas entre programas de televisión y medios impresos condujeron a mayor atención de los públicos, a un mejor recuerdo del mensaje y a una mayor credibilidad del mensaje, lo que generó, en definitiva, actitudes más positivas hacia la promoción en comparación con el uso de promociones repetitivas en un solo soporte. Crossmedia y cross-promotion son conceptos directamente relacionados porque se trata de la adaptación de las piezas a las diferentes plataformas de distribución para ganar más audiencia a través de la promoción cruzada entre ellas y mejorar, en consecuencia, los beneficios empresariales (Sixto, 2018b).

Con el auge de los medios digitales y del marketing de contenidos, las empresas cada vez utilizan más narrativas en sus estrategias (Bonnin y Rodriguez, 2019; Coombs, 2019). El storytelling siempre ha estado presente en nuestra vida y en todos los ámbitos de manera casi imperceptible, pero eso no implica que no 
deba considerarse una herramienta básica y de alta importancia para la gestión de la comunicación (Robledo-Dioses, Atarama-Rojas y López-Hermida, 2019). Una de las experiencias más recurridas son las narrativas transmedia, que se refieren a una nueva estética que surgió como respuesta a la convergencia de medios y que implica nuevas exigencias para los consumidores porque exigen la participación activa de las comunidades (Jenkins, 2009). Las narrativas transmedia suponen que el relato informativo se expanda por varias plataformas o medios de comunicación a través de piezas interrelacionadas que, a su vez, mantienen independencia narrativa y poseen sentido pleno. Por tanto, exigen una participación activa por parte del usuario para comprender la integridad del relato, algo que en muchos casos se produce gracias a la hipertextualidad, que permite y facilita el camino hacia otros espacios independientes y a la vez relacionados.

El periodismo incentiva la colaboración de los públicos en la producción de la narrativa y en el acto de compartir informaciones en el escenario digital (Silva y Damasceno, 2019), de modo que el relato se extiende de forma interrelacionada a través de múltiples medios, soportes o plataformas, por lo que se exige al usuario que visite varios para comprender el significado de la historia o al menos para obtener una perspectiva global. Al producirse un fraccionamiento intencionado de los contenidos cada medio aporta una parte específica y complementaria del relato, de tal forma que se haría necesario visualizar todas las partes para alcanzar el conocimiento integral del storytelling. Al usuario se le erradica la pasividad y se le facilita, en cambio, la elección del itinerario que desea realizar y, por otra parte, la posibilidad de intervenir en la historia mediante la interacción con la comunidad e, incluso a veces, a través de la cocreación de contenidos, lo que supone una modificación del storyboard original al permitir a las audiencias coconstruir su propia experiencia en su propio contexto mediante espacios participativos que permiten prácticas personalizadas y diferentes para cada uno de los usuarios que participan en ellas (Aitamurto, 2013; Prahalad y Ramaswamy, 2004). La tecnología, las redes sociales y movimientos como la gamificación, la social TV o la generalización en el uso de la segunda o de varias pantallas (multiscreen) han contribuido a enriquecer el discurso y a facilitar la construcción de estrategias transmedia (Rivera, 2012).

\section{Materialy métodos}

Desde mediados de la década de 1990 los cibermedios forman parte del ecosistema mediático, pues se multiplicaron de forma exponencial, de tal forma que lo que en un principio constituía una corriente o una tendencia se afianzó hasta alcanzar estándares punteros (Limia, López, Pereira, 2010). Comprobada la relevancia de los cibermedios en el contexto mediático actual, para esta investigación hemos seleccionado cinco ejemplos europeos significativos por la fuerza con que se adentraron en el escenario digital y la novedad que supusieron en el momento: eldiario.es (España), Mediapart.fr (Francia), observador.pt (Portugal), niusdiario.es (España) y Decorrespondent.nl (Holanda).

Aparte de los criterios expuestos, fundamentados en una observación directa (Sierra, 2007) del panorama comunicativo actual, la selección de la muestra se justifica también en términos de audiencia y conocimiento de marca, según los datos recogidos en Digital News Report 2019 (Reuters Institute, 2019). En terminología metodológica, se trata de una muestra de carácter no probabilístico, una técnica que se utiliza en escenarios de población pequeña y variable, lo que hace plausible seleccionar casos singulares o ejemplos característicos de ese universo (Igartua, 2006; Otzen y Manterola, 2017). Por otra parte, la pertinencia de los análisis de casos como recurso metodológico en Ciencias Sociales ha sido confirmado en investigaciones previas (Peña, 2009; Forni, 2010).

Tras la revisión de la bibliografía existente, establecimos los siguientes objetivos generales y específicos de estudio:

1. Examinar el uso de posibles acciones crossmedia y adaptación móvil en los cibermedios.

1.1 Verificar la existencia de presencias en redes sociales y en servicios de mensajería instantánea y analizar su tipología.

1.2 Determinar la ratio followers/following.

1.3 Identificar apps.

2. Determinar la existencia de estrategias cross-promotion para la difusión multiplataforma.

2.1 Comprobar la presencia de widgets en las webs corporativas.

2.2 Explorar técnicas destinadas a la promoción cruzada.

3. Detectar prácticas de narrativas transmedia en la distribución de contenidos.

3.1 Verificar el responsive design.

3.2 Indagar acerca de espacios destinados a la participación ciudadana y la cocreación de contenidos. 


\subsection{Localizar técnicas de swipe up y empleo de email marketing.}

Para ello hemos utilizado una metodología de carácter fundamentalmente exploratorio apoyada en técnicas de rastreo de webs, apps, correo electrónico y redes sociales. El rastreo se fundamentó en la localización de los ítems señalados en los objetivos de investigación, es decir, presencias en redes sociales y servicios de mensajería instantánea, carácter de dichas presencias, identificación de aplicaciones móviles, ubicación de widgets en páginas corporativas, análisis del diseño responsive, identificación de espacios destinados a la participación ciudadana e indagación de modelos asociados a narrativas transmedia.

Los datos cuantitativos procedentes de redes sociales se extrajeron un mismo día ( 27 de noviembre de 2019) para evitar errores de discriminación entre unas presencias y otras, aunque el examen analítico abarca hasta los dos últimos años. Para la evaluación del contenido autodestruible se utilizaron técnicas cualitativas de análisis de contenido, en especial para localizar la técnica de swipe up, que permite vincular la historia con otro espacio que se promociona desde la red social pero que es ajeno a ella.

\section{Resultados de investigación}

Todos los medios nativos digitales seleccionados hacen difusión de sus contenidos en las redes sociales, en concreto a través de Facebook, Twitter, Instagram, YouTube y Linkedln. Excepto Linkedln, que es una red vertical enfocada a asuntos profesionales y laborales, las demás son redes horizontales generalistas, enfocadas a un público no especializado. Sin embargo, detectamos una singularidad referente a la relación entre la difusión crossmedia y la arquitectura web de los sitios corporativos: todos los cibermedios presentan en sus webs los widgets de acceso a Facebook, Twitter e Instagram, pero ocultan los de YouTube y Linkedln, salvo eldiario.es y Observador en el primer caso y Observador y De Correspondent en el segundo. Tras un trabajo exploratorio en redes se descubre que los demás medios también cuentan con presencia en Linkedln y YouTube a pesar de no publicitarlo en sus webs oficiales, por lo que se constata la existencia de estrategias cross-promotion para publicitar algunas redes, pero no todas en las que figuran registros. Con respecto a los servicios de mensajería instantánea, solo se detecta una presencia en Telegram (eldiario.es) y otra en WhatsApp (Observador), ambas anunciadas mediante acciones cross-promotion en los respectivos portales (Tabla 1 ).

Las presencias en Facebook corresponden a páginas, el tipo de presencia adecuado para marcas y organizaciones, y todas ellas han sido verificadas por la red social mediante check azul, de modo que el usuario tiene la garantía de que se trata de las presencias oficiales de los medios. No se aprecian diferencias significativas entre el número de fans y de seguidores, aunque estos últimos alcanzan una cifra mayor en todos los casos (Tabla 2), de modo que hay más personas que reciben en su sección de noticias los contenidos publicados por los cibermedios que fans. Este fenómeno se explica porque a esos usuarios les interesa recibir en su espacio personal informaciones publicadas por los medios, pero públicamente no muestran a la comunidad que esa página les 'gusta'.

En Twitter todas las cuentas están confirmadas mediante la insignia azul de verificación y se aprecia en todos los casos una gran diferencia entre el número de followers y following, ya que estos últimos son irrelevantes en relación con los primeros. No se confirma, por tanto, que los medios realicen un proceso de follow-to-follow, sino que apuestan por la filosofía de que es un gran número de seguidores y un bajo número

\begin{tabular}{|c|c|c|c|c|c|c|c|}
\hline & \multicolumn{5}{|c|}{ Redes sociales } & \multicolumn{2}{|c|}{ Servicios de mensajería } \\
\hline & Facebook & Twitter & Instagram & YouTube & Linkedln & Telegram & WhatsApp \\
\hline $\begin{array}{c}\text { De } \\
\text { Correspondent }\end{array}$ & $x$ & $x$ & x & * & $x$ & & \\
\hline eldiario.es & $x$ & $x$ & $x$ & $x$ & * & $x$ & \\
\hline Mediapart & $x$ & $x$ & $x$ & * & * & & \\
\hline Nius & $x$ & $x$ & $x$ & * & * & & \\
\hline Observador & $x$ & $x$ & $x$ & $x$ & $x$ & & $x(1)$ \\
\hline \multicolumn{8}{|c|}{ X significa presencia cross-promotion desde web. } \\
\hline significa presen & cia crossme & a mediar & astreo manu & & & & \\
\hline
\end{tabular}

Tabla 1. Presencias corporativas de los cibermedios en redes sociales y servicios de mensajería. Elaboración propia. 
de seguidos lo que confiere relevancia a las cuentas organizacionales, más cuando desde Twitter no se establecen límites en cuanto al número de cuentas que se pueden seguir, sino que se permiten hasta 400 seguimientos diarios en cuentas nuevas y 1.000 en cuentas verificadas ${ }^{1}$. De hecho, al calcular la ratio de following entre followers los resultados sobrepasan las centésimas $(<0,01)^{2}$ salvo en el caso de Nius (tabla 3$)$, lo que no implica un cambio de tendencia en el follow back, sino la juventud de un proyecto que fue lanzado en septiembre de 2019.

Esta postura por mantener cuentas relevantes e influyentes que generen tendencias y corrientes de opinión se reproduce también en Instagram, donde todos los medios mantienen una ratio $<0,005$ (tabla 3), excepto Nius, aunque eso no implica un cambio de tendencia, sino simplemente un número inferior de seguidores, algo que no se traduce en una mengua significativa de la influencia. Sin embargo, sí que se diagnostica una preferencia notoria por preferir Twitter a Instagram para la difusión crossmedia de los contenidos, de modo que la media de informaciones trasladadas a Twitter se sitúa en 105.840, mientras que en Instagram la cifra desciende a 940. Este último dato es superado por YouTube, donde se albergan una media de 1.908,6 vídeos, lo que refleja un fuerte desafío por los contenidos audiovisuales, que tienen gran acogida entre los públicos, pues logran 21.979 .000 visualizaciones de media.

Instagram solo ha validado las cuentas de Nius y Observador, a pesar de que esta red sí permite el envío de la solicitud de verificación por parte de la marca anexando la documentación oficial de acreditación de identidad y justificando la conexión con la página de Facebook, si bien la decisión final siempre corresponde a la red social. Con todo, Instagram no es la red preferida por las audiencias para informarse -quizás debido a la juventud de su target-, sino que Twitter y Facebook son las redes que concentran más seguidores, mientras que
Linkedln y YouTube son las que menos (Tabla 2), a pesar de que también en ambos casos las presencias organizacionales son las pertinentes para las marcas, esto es, canal en el primer caso y página en el segundo.

\begin{tabular}{|r|l|l|}
\cline { 2 - 3 } \multicolumn{1}{c|}{} & \multicolumn{1}{|c|}{ Twitter } & \multicolumn{1}{c|}{ Instagram } \\
\hline De Correspondent & 0,000349 & 0,00264 \\
\hline eldiario.es & 0,000477 & 0,00011 \\
\hline Mediapart & 0,00072 & 0,00025 \\
\hline Nius & 0,01707 & 0,01048 \\
\hline Observador & 0,00033 & 0,00043 \\
\hline
\end{tabular}

Tabla 3. Ratios following/followers en Twitter e Instagram. Elaboración propia.

Con respecto a los servicios de mensajería instantánea, Observador ofrecía a los usuarios la posibilidad de agregar su contacto de WhatsApp para remitirles contenidos, aunque esta opción fue eliminada durante el transcurro de esta investigación. Enviaban seis informaciones diarias -tres matutinas y tres vespertinas- y lo hacían desde una cuenta personal, no de WhatsApp Business, la opción específica que ofrece la aplicación para marcas y organizaciones y que permite otras opciones comunicativas para la gestión de la comunicación como el establecimiento de horarios de atención al público, geolocalización, enlace directo para storytelling transmedia mediante otras plataformas como el correo electrónico, automatización de respuestas, clasificación y jerarquización de la información a través de un sistema de etiquetaje y destacado de mensajes, verificación de cuenta o acceso a datos estadísticos de audiencia y reporting. Se trataba, en cualquier caso, de un servicio unidireccional en que el medio solo enviaba, pero no contestaba a las inquietudes de los usuarios ni practicaba ningún tipo de interacción a pesar de tener activo el check azul de confirmación de lectura. eldiario.es, en cambio, opta por un canal de Telegram para la difusión crossmedia de contenidos periodísticos que

\begin{tabular}{|c|c|c|c|c|c|c|c|c|c|c|c|c|}
\hline & \multicolumn{12}{|c|}{ Redes sociales } \\
\hline & \multicolumn{2}{|c|}{ Facebook } & \multicolumn{3}{|c|}{ Twitter } & \multicolumn{3}{|c|}{ Instagram } & \multicolumn{3}{|c|}{ YouTube } & \multirow{2}{*}{ 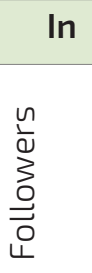 } \\
\hline & $\stackrel{\otimes}{\stackrel{y}{\exists}}$ & 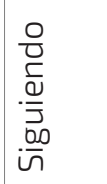 & $\begin{array}{l}\frac{n}{1} \\
\frac{1}{2} \\
\stackrel{0}{0} \\
\stackrel{0}{0} \\
\stackrel{1}{1}\end{array}$ & 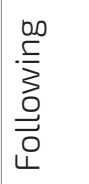 & 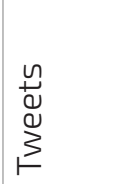 & $\begin{array}{l}\frac{n}{0} \\
\frac{1}{2} \\
\frac{0}{0} \\
\stackrel{0}{0} \\
\stackrel{1}{1}\end{array}$ & 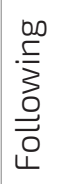 & 官 & $\begin{array}{l}u \\
\text { Ũ } \\
\text { v }\end{array}$ & 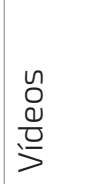 & $\begin{array}{l}\dot{\pi} \\
\stackrel{\sim}{J} \\
\stackrel{.}{>}\end{array}$ & \\
\hline De Corres. & $211,5 \mathrm{~K}$ & $212 K$ & $185,8 \mathrm{~K}$ & 65 & $17,1 \mathrm{~K}$ & $14 K$ & 37 & 79 & $2,8 \mathrm{~K}$ & 42 & $165 \mathrm{~K}$ & $24 K$ \\
\hline eldiario.es & $535 K$ & $541 \mathrm{~K}$ & $1 \mathrm{M}$ & 477 & $225,6 K$ & $83,8 \mathrm{~K}$ & 95 & 736 & $67,6 \mathrm{~K}$ & $5,2 \mathrm{~K}$ & $40,1 \mathrm{M}$ & $7,4 \mathrm{~K}$ \\
\hline Mediapart & 1,09M & $1,1 \mathrm{M}$ & $2,5 \mathrm{M}$ & $1,8 \mathrm{~K}$ & $92 \mathrm{~K}$ & $186 \mathrm{~K}$ & 474 & $1,2 \mathrm{~K}$ & $273 K$ & $2,3 \mathrm{~K}$ & $56 \mathrm{M}$ & $18,2 \mathrm{~K}$ \\
\hline Nius & $8 \mathrm{~K}$ & $9 K$ & $11,3 \mathrm{~K}$ & 193 & $6 K$ & $4,1 \mathrm{~K}$ & 43 & 185 & $2,3 \mathrm{~K}$ & 101 & $630 K$ & 202 \\
\hline Observardor & $776,3 \mathrm{~K}$ & $795 K$ & $113,9 \mathrm{~K}$ & 38 & $188,5 \mathrm{~K}$ & $127 \mathrm{~K}$ & 55 & $2,5 \mathrm{~K}$ & $35,5 K$ & $1,9 \mathrm{~K}$ & $13 \mathrm{M}$ & $40 K$ \\
\hline
\end{tabular}

Tabla 2. Diagnóstico de la presencia en redes sociales de los cibermedios. Elaboración propia. 
figuran publicados en la web. El canal cuenta con 26,1K suscriptores y realizan una media de dos envíos diarios, que son visualizados por una media de $13,5 \mathrm{~K}$ de usuarios. Como todos los canales de Telegram se trata de un sistema unidireccional en que el usuario asume un rol pasivo de receptor, pero que desde el punto de vista corporativo supone otra puerta de entrada a los contenidos porque genera gran tráfico desde el móvil hacia la web mediante urls. En ambos casos este modelo de difusión exige al usuario recurrir a otro espacio web para completar el relato, por lo que podemos referirnos al empleo de narrativas transmedia.

Ni Nius ni De Correspondent ofrecen app para móviles, mientras que el resto de diarios analizados -eldiario. es, Mediapart y Observador-facilitan tanto la versión para Android como para iPhone. Sin embargo, todas las presencias se presentan en diseño responsive 0 adaptivo, una técnica de diseño web que permite la visualización correcta de una misma página en dispositivos distintos, generalmente los tres contemplados en el análisis: ordenador (de escritorio o portátil), tableta y móvil (Tabla 4). Al utilizar este tipo de diseño los elementos de la web se redimensionan y colocan de forma que se adaptan al ancho de cada dispositivo, lo que reduce los tiempos de desarrollo, evita la duplicidad de contenidos y aumenta la viralidad cross-content al permitir compartir de forma más rápida e intuitiva, por lo que, en definitiva, se mejora la experiencia del usuario. Los contenidos que se ofrecen en app son los mismos que en las webs, de modo que se efectúa una distribución crossmedia de las informaciones, que son idénticas en contenido, pero adaptadas tecnológica- mente a los diferentes soportes, aunque tanto eldiario. es como Observador permiten personalizar la app para recibir informaciones a demanda. Por otra parte, se aprecian técnicas de cross-promotion en las webs de todos los cibermedios, ya que anuncian la app para que los usuarios las descarguen en sus móviles.

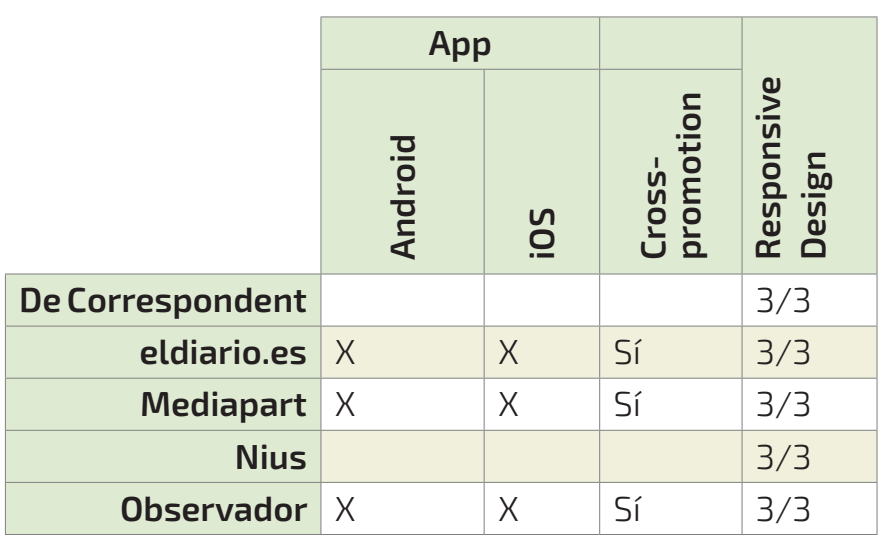

Tabla 4. Apps y diseño responsive en los cibermedios. Elaboración propia.

Todos los cibermedios, excepto Nius, concentran gran parte del esfuerzo de sus estrategias de cross-promotion en la habilitación de espacios call to action destinados a la captación de socios (téngase en cuenta que De Correspondent y Mediapart no albergan publicidad). De hecho, la mayoría restringen ciertos contenidos para sus abonados, aunque donde más patente se hace la diferencia entre usuarios sin registrar y asociados es en las posibilidades ofrecidas para la cocreación. Nius no permite el empoderamiento de los receptores para la cocreación de contenidos más allá de las interacciones sociales que puedan derivarse de la participación

\begin{tabular}{|c|c|c|c|}
\hline & Usuarios sin registrar & Usuarios registrados & Socios \\
\hline $\begin{array}{l}\text { De } \\
\text { Correspondent }\end{array}$ & - Sin acceso a contenidos. & $\begin{array}{l}\text { - Propuestas de cocrea- } \\
\text { ción de contenidos. }\end{array}$ & $\begin{array}{l}\text {-Colaboración con socios expertos } \\
\text { en temas. } \\
\text { - Involucración en investigaciones. } \\
\text { - Lectores como fuente de valor. }\end{array}$ \\
\hline eldiario.es & $\begin{array}{l}\text { - Acceso total a contenidos. } \\
\text { - Filtraciones confidenciales. }\end{array}$ & $\begin{array}{l}\text { - Corrección de textos. } \\
\text { - Interacciones sociales. } \\
\text { - Personalización temas } \\
\text { en app. }\end{array}$ & $\begin{array}{l}\text { - Comentarios tenidos en cuenta por } \\
\text { la redacción. } \\
\text { - Encuentros específicos. } \\
\text { - Votaciones online }\end{array}$ \\
\hline Mediapart & $\begin{array}{l}\text { - Sin acceso a contenidos } \\
\text { (solo cocreados). } \\
\text { - Propuestas de temas y ugc. } \\
\text { - Filtraciones confidenciales. }\end{array}$ & / & $\begin{array}{l}\text { - Club para la cocreación. } \\
\text { - Blog y espacio participativo. }\end{array}$ \\
\hline Nius & $\begin{array}{l}\text {-Acceso en abierto a todos } \\
\text { los contenidos. }\end{array}$ & - Interacciones sociales. & l \\
\hline Observador & $\begin{array}{l}\text { - Acceso parcial a contenidos. } \\
\text { - Corrección de texto. } \\
\text { - Envío de ugc. }\end{array}$ & $\begin{array}{l}\text { - Interacciones sociales. } \\
\text { - Alertas. } \\
\text { - Temario personalizado } \\
\text { en app. }\end{array}$ & $\begin{array}{l}\text { - Se añade acceso y participación en } \\
\text { contenidos Premium. }\end{array}$ \\
\hline
\end{tabular}

Tabla 5. Posibilidades de cocreación de contenidos en los cibermedios. Elaboración propia. 
en la comunidad a partir de un contenido determinado, mientras que en los demás las opciones para cocrear aumentan en función de la vinculación del usuario con el medio, de modo que las más atractivas se reservan para usuarios registrados y socios (Tabla 5).

En ningún caso se comprueba la existencia de contenidos creados ex profeso para publicar en redes sociales, sino que se realiza una difusión cross-content de las informaciones de la web, pero adaptadas a los lenguajes específicos de cada plataforma. Salvo De Correspondent, todos los medios realizan una transmisión continua de contenidos en Facebook y Twitter, lo que supone que cada una o dos horas se traslada una información nueva a estas redes. En De Correspondent las publicaciones se limitan a aproximadamente una al día y presentan la salvedad de que permiten visualizar contenidos que en la web no se ofrecen en abierto, algo que no sucede en Mediapart, donde al redirigir al usuario hacia la web vuelve a encontrarse con la restricción de contenidos específicos para socios.

A pesar de que a priori Instagram pudiera no parecer la red más adecuada para la distribución de contenidos periodísticos debido a su limitación textual y a la presentación de posts únicamente visuales o audiovisuales, lo cierto es que Mediapart, Nius y Observador la utilizan para difusión continua al igual que Facebook y Twitter, mientras que eldiario.es realiza difusiones diarias y $D e$ Correspondent de manera más esporádica. Todos los medios realizan descripciones largas de las fotografías o vídeos, lo que exige al usuario hacer un nuevo clic para 'ver más'. Estas descripciones aparecen siempre acompañadas de hashtags, un recurso lingüístico propio de esta red, salvo en el caso de Observador, que, por otra parte, presenta la singularidad de emplear una técnica transmedia en todas las descripciones, ya que propone al usuario la alternativa de completar el relato accediendo al link corporativo que figura en la bio. En Twitter el uso de hashtags es más limitado, a pesar de tratarse de un formato narrativo que se originó en esta red, y solo los emplean a veces eldiario.es y Mediapart. En cambio, es común la mención @ que permite identificar la autoría de la pieza periodística, lo que no implica necesariamente la existencia de una narrativa transmedia, pero sí la posibilidad de poder establecer un contacto entre usuario y periodista.

La participación en YouTube es menos frecuente, en especial en el caso de De Correspondent, donde pueden encontrarse periodos de dos meses sin subir un vídeo nuevo. Las frecuencias de actualización varían entre los 2-3 vídeos por semana en eldiario.es y Mediapart y los 7-10 vídeos semanales en Observador y Nius. Todos los cibermedios estructuran los vídeos en listas de repro- ducción y sobresale el número de emisiones en live que realiza Nius. En Linkedln las publicaciones se realizan de forma esporádica en esos periódicos, mientras que Mediapart también utiliza esta red para hacer difusión continua de los contenidos. De Correspondent y eldiario. es la emplean con una frecuencia similar a YouTube.

De los espacios en que los cibermedios tienen presencia y que permiten la emisión de contenido autodestruible, es decir, WhatsApp, Instagram, Facebook y YouTube, solo se confirma la existencia de historias en Instagram y Facebook. Para Nius y Observador este modelo narrativo forma parte de su quehacer diario y en ambos casos se recurre a swipe up para completar el relato informativo en la web corporativa, por lo que nos encontramos ante un nuevo caso de narrativa transmedia. Nius apuesta por la fragmentación en varias historias de una misma pieza, generalmente un reportaje, y Observador opta por la difusión de historias consecutivas que no están interrelacionadas y que presentan un diseño dinámico. En estos dos medios se recurre diariamente a este tipo de narrativa, mientras que en De Correspondent, eldiario.es y Mediapart la difusión se realiza de manera de forma ocasional, si bien es cierto que en todos los perfiles de Instagram se presentan historias destacadas -aquellas que permanecen fijadas en el perfil sin desaparecer a las 24 horas $^{3}$ - organizadas por temáticas.

Por último, otro de los mecanismos que ofrecen los espacios digitales para la difusión de contenidos es el email marketing, que en el caso de los cibermedios se materializa en el envío de una newsletter a una base de datos conformada por usuarios previamente suscriptos, de modo que se utiliza como una estrategia de marketing directo para crear lealtad entre los usuarios y generar tráfico hacia la web. Salvo De Correspondent, que solo envía boletines comerciales, todos los cibermedios emplean esta técnica con finalidad editorial para trasladar un resumen de los contenidos-incluso personalizados, como sucede en Observador- a los públicos, pero que necesariamente se ven obligados a completar

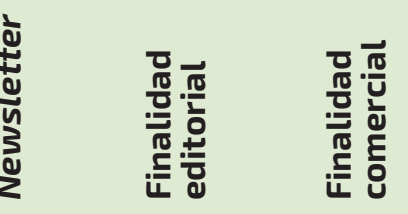

De Correspondent Sí

\begin{tabular}{r|l|l|l|}
\cline { 2 - 4 } eldiario.es & Sí & $X$ & $X$ \\
Mediapart & Sí & $X$ & $X$ \\
\cline { 2 - 4 } Nius & Sí & $X$ & \\
\cline { 2 - 4 } Observador & Sí & $X$ &
\end{tabular}

Tabla 6. Empleo del email marketing en los cibermedios y finalidad. Elaboración propia. 
el relato informativo en las webs corporativas, por lo que podemos referirnos a hipertextualidad vinculada a narrativa transmedia (Tabla 6).

\section{Conclusiones finales}

Los cibermedios emplean cuatro espacios digitales para la difusión crossmedia de los contenidos que publican: las redes sociales, los servicios de mensajería instantánea, las aplicaciones móviles y el email marketing. En todos los casos se diagnostica una distribución cross-content que no acarrea producción de contenidos nuevos para cada soporte, sino adaptación de las piezas a la distribución multiplataforma, empleo de los lenguajes específicos y ajuste tecnológico basado en un diseño responsive para posibilitar la visualización de contenidos en pantallas diferentes y a la vez (multiscreen).

Existe coincidencia entre las redes donde más seguidores concentran los cibermedios y las que utilizan para difundir contenidos, en particular Facebook y Twitter, donde la tendencia es distribuir contenidos de forma continuada. Las frecuencias de difusión disminuyen en Instagram, YouTube y Linkedln, por este orden, sin que se aprecie desatención a estas redes en ningún caso. Sin embargo, las redes sociales se utilizan más como un escenario de difusión de contenidos que como un espacio dialógico, lo que refleja más interacción de los públicos que de los medios, que apenas intervienen en la comunidad de usuarios. Esta tendencia se reproduce en los servicios de mensajería instantánea como Telegram, que se emplea únicamente de forma unidireccional sin ofrecer al usuario la posibilidad de plantear preguntas o participar en un diálogo, por lo que, en realidad, se utiliza a modo de newsletters y no como escenarios conversacionales verdaderos.

Se aprecia explotación de las estrategias de cross-promotion en todos los cibermedios, pues existe una fuerte apuesta por conducir al usuario de unas plataformas a otras, esto es, de la web a las redes, de las redes a la web, de los boletines electrónicos a la web, de los servicios de mensajería instantánea a la web y a las redes, y de unas redes a otras. La finalidad con que se implementan estas estrategias responde, por tanto, a un aumento del tráfico, lo que se traduce en más audiencia y consecuentemente en beneficio empresarial. En todo este proceso de derivación de unos espacios a otros se constata la existencia de narrativas transmedia que están más vinculadas a la hipertextualidad que a la fragmentación del storytelling en varios espacios. En este sentido, tanto las newsletters como las historias efímeras de Instagram o Facebook se emplean como recursos call to action que mediante técnicas como swipe up exigen acceder a otro espacio digital para obtener una visión integral del relato informativo.

En resumen, y a modo de conclusión, es evidente que los cibermedios no limitan la distribución de su producción informativa a los espacios corporativos, sino que emplean los principales recursos interactivos y de distribución que habilita la web 2.0 para realizar una difusión crossmedia de sus productos informativos. No se trata de espacios excluyentes, sino complementarios, que se refuerzan unos a otros, lo que explica en parte que no se preparen piezas exclusivas para cada espacio y que se opte por una adaptación a los lenguajes y estilos de cada soporte. En el caso de los móviles la adaptación es simplemente tecnológica, de modo que se procura una visualización acomodada al tamaño de la pantalla, pero los contenidos son idénticos a los accesibles desde otro dispositivo fijo o portátil. Narrativas cross-content, técnicas de cross-promotion y narrativas transmedia vinculadas a la hipertextualidad se usan de manera conjunta para satisfacer las necesidades de unas audiencias cada vez más amoldadas a navegar de un sitio digital a otro a través de dos o más pantallas $y$, por otra parte, para hacer viables iniciativas periodísticas nacidas en y para Internet, algunas sin financiación publicitaria y dependientes únicamente de las aportaciones de los socios, donde se hace imprescindible la adaptación al necesario convergente tanto desde los puntos de vista empresarial y tecnológico, como de los contenidos y los profesionales de la información.

\section{Notas a final de página}

1 Además de los límites diarios, existen tasas de seguimiento que entran en vigencia al seguir un número determinado de cuentas. Una cuenta puede seguir por defecto otras 5.000 hasta tener más seguidores, aunque esa cifra es diferente para cuenta y la calcula Twitter automáticamente en base a la proporción entre seguidores y seguidos.

2 Una cuenta con una ratio superior a 1 se considera que no es relevante. Entre 0,5 y 1 puede ser aceptable, aunque siempre es preferible situarse por debajo de los 0,5 puntos. Una ratio inferior a 0,2 implica que la cuenta tiene influencia y que marca tendencia y corrientes de opinión. Las cuentas con una ratio que no supere los 0,1 puntos son ya influencers destacables y las que presentan un resultado menor a 0,005 puntos corresponden a marcas personales de personajes públicos o a marcas organizacionales muy conocidas (Sixto, 2018a).

3 Las historias de YouTube desaparecen a la semana de su publicación.

\section{Financiación}

Este texto está elaborado en el marco del proyecto Cibermedios nativos digitales en España: formatos narrativos y estrategia móvil (RTI2018-093346-B-C33), del Ministerio de Ciencia, Innovación y Universidades. 
Este proyecto está cofinanciado por el fondo estructural FEDER.

\section{Referencias}

Aitamurto, T. (2013). Balancing Between Open and Closed: Cocreation in Magazine Journalism. Digital Journalism, 1(2), 229-251. https://doi.org/10.1080/21670811.2012.750150

Bonnin, G., y Rodríguez, M. (2019). The narrative strategies of B2B technology brands. Journal of Business \& Industrial Marketing, 34(7), 1448-1458. https://doi.org/10.1108/JBIM-03-2019-0112

Castells, M. (2000). La Sociedad Red. Alianza Editorial.

Coombs, T. (2019). Transmedia storytelling: a potentially vital resource for CSR communication. Corporate Communications: An International Journal, 24(2), 351-367. https://doi.org/10.1108/CCIJ-112017-0114

Copple, E. (2012). "Affluencers" by Bravo: Defining an audience through cross-promotion. Popular Communication, 10(4), online. https://doi.org/10.1080/15405702.2012.715327

Dailey, L., Demo, L., y Spillman, M. (2010). The Convergence Continuum: A model for studying colaboration between media newsrooms. Atlantic Journal of Communication, 13(3), 150-168. https://doi. org/10.1207/s15456889ajc1303_2

Doyle, G. (2002). Media ownership: The economics and politics of convergence and concentration in the UK and European media. Sage.

Forni, P. (2010). Los estudios de caso: Orígenes, cuestiones de diseño y sus aportes a la teoría social. Miríada. Investigación en Ciencias Sociales, 3(5), en línea. https://p3.usal.edu.ar/index.php/miriada/ article/view/5/153

Franquet, R., y Villa, M. I. (2012). Interpretation and analysis of cross media content. The case of Televisió de Catalunya. Anàlisi: Quaderns de comunicació i cultura, extra 1, 49-63. https://doi.org/10.7238/a. v0im.1501

García Avilés, J. A. (2006). Desmitificando la convergencia periodística. Chasqui: Revista Latinoamericana de Comunicación, (94), 34-39.

Golding, P., y Murdock, G. (1966). Culture, Communications and Political Economy. En J. Curran y M. Gurevitch (Ed.), Mass Media and Society. Arnold.

Gordon, R. (2003). The meanings and implications of Convergence. En K. Kawamoto (Ed.), Digital Journalism: Emerging media and changing horizons of Journalism. Rowman \& Littlefield Publishers.

Igartua, J. J. (2006). Métodos cuantitativos de investigación en Comunicación. Bosch.

Jenkins, H. (2004). The cultural logics of media convergence. International Journal of Cultural Studies, 7(1), 33-43.

Jenkins, H. (2006). Convergence Culture. Where old and new media collide. New York University Press.

Jenkins, H. (2009). Cultura da convergência. Aleph.

Ketterer, S., Weir, T., Smethers, S., y Beck, J. (2004). Case study shows limited benefits of convergence. Newspaper Research Journal, 25(3), 52-65.

Kjus, Y. (2014). Impact of prestige programs on production practices: the case of crossmedia and audience participation in public service organizations. Journal of Media Practice, 10(2-3), online. https://doi. org/10.1386/jmpr.10.2-3.167_1

Limia, M., López, X., y Pereira, X. (2010). 15 años de cibermedios en España. En X. López y X. Pereira (Ed.). Convergencia Digital. Reconfiguración de los medios de comunicación en España, (pp. 9-25). Universidad de Santiago de Compostela.

Lowrey, W., Daniels, G., y Becker, L. B. (2005). Predictors of convergence curricula in Journalism and Mass Communication programs. Journalism and Mass Communication Educator, (60), 32-46.

Ma, Q., Tanaka, K., y Nadamoto, A. (2006). Complementary information retrieval for crossmedia news content. Information systems, 31(7), 659-678. https://doi.org/10.1016/j.is.2005.12.004

Martínez-Sala, A. M., y Campillo Alhama, C. (2018). Video as support in the digital narrative of 2.0 tourist events. Miguel Hernández Communication Journal, (9), 227-260. https://doi.org/10.21134/mhcj.v0i9.230

Martínez-Sala, A. M.; Segarra-Saavedra, J., y Monserrat-Gauchi, J. (2018). Los millennials como prosumers y adprosumers en las redes sociales corporativas. Cuadernos.info, (43), 137-159. https://doi. org/10.7764/cdi.43.1335

Masip, P., y Micó, J. Ll. (2009). El periodista polivalent en el marc de la convergència empresarial. Quaderns del CAC, (31-32), 85-92.

Negroponte, N. (1995). Being digital. A. A. Knopf.

Otzen, T., y Manterola, C. (2017). Sampling Techniques on a Population Study. International Journal of Morphology, (35), 227-232.

Peña, W. (2009). El estudio de caso como recurso metodológico apropiado a la investigación en Ciencias Sociales. Educación y Desarrollo Social, 3(2), 180-195.

Prahalad, C. K., y Ramaswamy, V. (2004). Co-creation experiences: the next practice in value creation". Journal of interactive marketing, 18(3), 5-14. https://doi.org/10.1002/dir.20015

Quinn, S. (2005). Convergent Journalism. The fundamentals of multimedia reporting. Peter Lang.

Rivera, D. (2012). ¿Qué es transmedia y storytelling? https://agencia. best/blog/transmedia-y-storytelling/

Robledo-Dioses, K., Atarama-Rojas, T., y López-Hermida, A. (2019). El storytelling como herramienta de comunicación interna: una propuesta de modelo de gestión. Revista Cuadernos de Gestión, versión online. https://doi.org/10.5295/cdg.170755kr

Reuters Institute (2019). Digital News Report 2019. Reuters Intitute https://www.digitalnewsreport.org/

Salaverría, R., y Negredo, S. (2008). Periodismo integrado: convergencia de medios y reorganización de redacciones. Sol90 Media.

Salaverría, R. (2010). Estructura de la convergencia. En X. López y X. Pereira (Ed.). Convergencia Digital. Reconfiguración de los medios de comunicación en España, (pp. 27-40). Universidad de Santiago de Compostela.

Salaverría, R., García Avilés, J. A., y Masip, P. (2010). Concepto de convergencia periodística. En X. López y X. Pereira (Ed.). Convergencia Digital. Reconfiguración de los medios de comunicación en España, (pp. 41-64). Universidad de Santiago de Compostela.

Segarra-Saavedra, J., y Tur-Viñes, V. (2017). Creatividad publicitaria: marcas vs prosumer amateur. La viralidad del spot de Eugen Merher para Adidas. En I. García-Medina y V. Tur-Viñes (Ed.), Diálogos bilaterales entre investigadores de la Glasgow Caledonian University (Reino Unido) y la Universidad de Alicante (España). Estudios interdisciplinares, (pp. 175-184). Colección Mundo Digital de Revista Mediter- 
ránea de Comunicación.

Sierra, R. (2007). Técnicas de investigación social: teoría y ejercicios. International Thomson

Silva, P. E., y Damasceno, M. (2019). As características transmídia e crossmídia do jornalismo audiovisual no instagram: uma análise da autorrepresentação de jornalistas da Globo News tv. En J. Canavilhas, C. Rodrigues y F. Giacormelli (Ed.). Narrativas jornalísticas para dispositivos móveis, (pp. 237-254).

Singer, J. B. (2006). Partnerships and public service: Normative issues for journalists in converged newsrooms. Journal of Mass Media Ethics, 21(1), 30-53.

Sixto, J. (2018a). Gestión profesional de redes sociales. Estrategias y rutinas de community management. Comunicación Social.

Sixto, J. (2018b). La radio española en las redes sociales: crossmedia en el escenario convergente. Communication Papers Media Literacy $\&$ Gender Studies, 7(14), 181-205. https://doi.org/10.33115/udg_bib/ cp.v7i14.22284

Tang, T., Newton, G., y Wang, X. (2007). Does Synergy Work? An examination of cross-promotion effects. International Journal on Media Management, 9(4), 127-134. https://doi. org/10.1080/14241270701632654

Villa, M. I. (2011). Producción crossmedia. El caso de Televisió de Catalunya (Tesis doctoral).

\section{CV}

José Sixto-García. Es profesor del departamento de Ciencias de la Comunicación de la Universidad de Santiago de Compostela y forma parte del grupo de investigación Novos medios (Gl-1641 NM). Entre 2013 y 2019 fue director del Instituto de Medios Sociales. Es doctor en Comunicación (Premio Extraordinario), Máster Universitario en Profesorado, MBA en Dirección y Gestión de Empresas y Licenciado en Periodismo (Premio Extraordinario). Sus líneas de investigación abarcan los contextos digitales, las redes sociales y las nuevas narrativas y su incidencia en la producción informativa. Su índice h es 13. Web del autor: https://www.researchgate.net/profile/Jose_Sixto_Garcia

Xosé López-García. Es catedrático de Periodismo en el Departamento de Ciencias de la Comunicación de la Universidad de Santiago de Compostela. Es doctor en Historia y periodista. Coordina desde el año 1994 el grupo de investigación Novos medios (Gl-1641 NM), que tiene entre sus líneas de investigación el estudio de los medios digitales e impresos, el análisis del impacto de la tecnología en la comunicación mediada, el análisis del funcionamiento y financiación de las industrias culturales. Su índice h es 21. Web del autor: https://www. researchgate.net/profile/Xose_Garcia

осм Observatorio de Cibermedios

\section{https://observatoriocibermedios.upf.edu/}

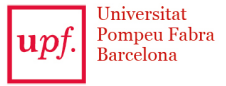

Departamento de Comunicación

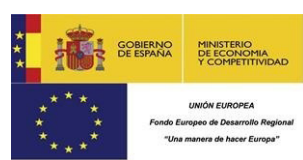

El Observatorio de Cibermedios es una producción del Grupo de Investigación en Documentación Digital y Comunicación Interactiva (DigiDoc) del Departamento de Comunicación de la Universitat Pompeu Fabra.

El Observatorio de Cibermedios (OCM) forma parte del proyecto del Plan Nacional "Narración interactiva y visibilidad digital en el documental interactivo y el periodismo estructurado". RTI2018-095714-B-C21 (MINECO/FEDER), Ministerio de Ciencia, Innovación y Universidades (España). 